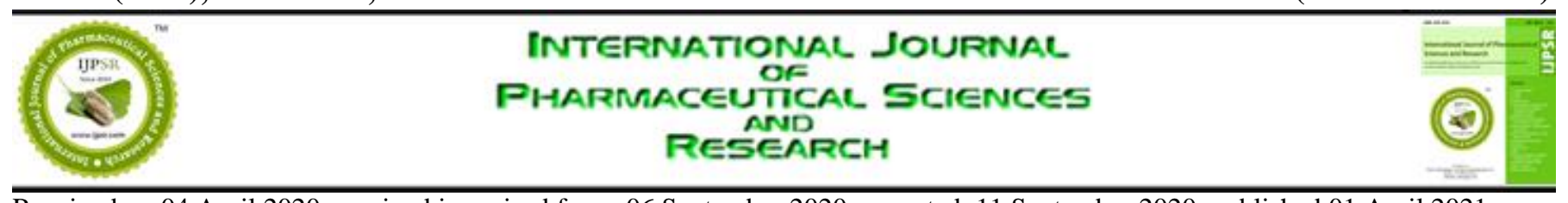

Received on 04 April 2020; received in revised form, 06 September 2020; accepted, 11 September 2020; published 01 April 2021

\title{
SYNTHESIS, CHARACTERIZATION AND BIOLOGICAL EVALUATION OF NOVEL QUINAZOLINONE DERIVATIVES AS ANTI-INFLAMMATORY AGENTS
}

Ritesh Patel $^{*}{ }^{1}$, Rohit Saraswat ${ }^{2}$ and Sujit Pillai ${ }^{3}$

Department of Pharmaceutical Chemistry ${ }^{1}$, Indore Institute of Pharmacy, Indore - 453331, Madhya Pradesh, India.

Department of Pharmacy ${ }^{2}$, OPJS University, Churu - 331303, Rajasthan, India.

GRY Institute of Pharmacy ${ }^{3}$, Khargone - 451228, Madhya Pradesh, India.

Keywords:

5-chloro anthranilic acid,

Quinazolinone, Anti-inflammatory activity, Diclofenac sodium

\section{Correspondence to Author:}

Ritesh Patel

Assistant Professor,

Department of Pharmaceutical

Chemistry, Indore Institute of

Pharmacy, Rau-Pithampur Road,

Indore- 453331, M.P., India

E-mail: riteshcop@gmail.com

\begin{abstract}
A series of quinazolinone derivatives were synthesized and screened for anti-inflammatory activity. 5-chloro anthranilic acid undergoes acetylation in the presence of acetic anhydride and anhydrous sodium acetate to give 5-chloro-N-acetyl anthranilic acid as intermediate-I which upon cyclization in the presence of phosphorous pentaoxide, glacial acetic acid, and para aminobenzoic acid to yield 4[6-chloro-2- methyl-4-oxoquinazolin -3(4H)-yl] benzoic acid as intermediate-II. This resulted in intermediate-II undergo mannich base reaction to produce novel quinazolinone derivatives $(\mathrm{Q} 1$ - Q16) on the reaction of formaldehyde with different aromatic amines. Sixteen different quinazolinone derivatives were synthesized. Structural assignments of these compounds have been made by elemental analysis, FTIR, $1 \mathrm{H} \mathrm{NMR,} \mathrm{and} \mathrm{mass} \mathrm{spectral} \mathrm{data.} \mathrm{Among} \mathrm{the}$ synthesized compounds Q3, Q8, and Q 15 showed high antiinflammatory activity against standard drug Diclofenac sodium. A majority of the tested compounds had shown good consequence to moderate anti-inflammatory activity.
\end{abstract}

INTRODUCTION: It is evident from the literature that, Quinazolinone is a heterocyclic compound play a vital role in synthetic, medicinal chemistry. The synthetic derivatives of quinazolinone are utilized as a therapeutic agent for combating against different pathological conditions. 5-chloro anthranilic acid is mainly employed for the synthesis of quinazolinone compounds as starting materials ${ }^{1}$.

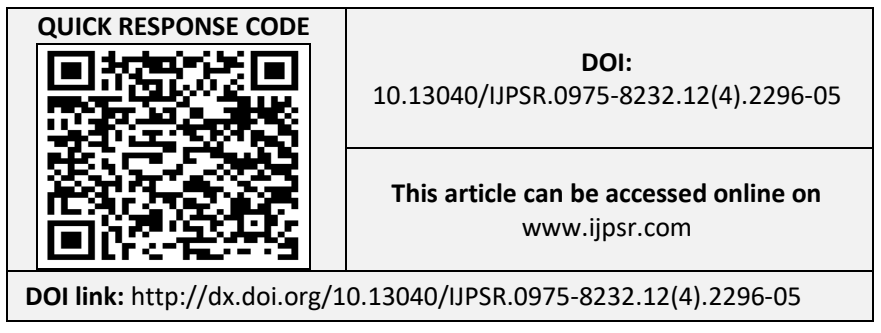

Quinazolinone and its derivatives possess a major class of biologically active compounds which exhibited a large spectrum of therapeutic activities, including; anti-malaria ${ }^{12}$, analgesic ${ }^{3}$, antioxidant ${ }^{4}$, anticancer ${ }^{5}$, antiviral ${ }^{16}$, anti-feedant ${ }^{7}$, sedativehypnotic ${ }^{8}$, anticonvulsant 9 , antimicrobial 10 , antialgal ${ }^{11}$, hypotensive ${ }^{12}$ and anti-inflammatory 13

Recently quinazolinone derivatives seek the great attention of researchers in organic and medicinal chemistry due to their prompt biological activities. Encouraged by the therapeutic diversity of quinazolinone containing moiety and the comparative ease of convertibility of anthranilic acid to quinazolinone, we took up the synthesis of certain novel quinazolinone from 5-chloro 
anthranilic acid and evaluated their antiinflammatory activity ${ }^{14}$.

\section{EXPERIMENTAL:}

Materials and Methods: All the chemicals used in the synthesis of the intermediates and final derivatives were of A.R grade and procured from the Merck and LOBA chemicals. All the synthesized quinazolinone derivatives were characterized by melting point determination using Veergo digital melting point apparatus in open capillary tubes and were uncorrected.

IR spectra were recorded using Perkin Elmer FTIR spectrophotometer using $\mathrm{KBr}$ pellets techniques, and ${ }^{1}$ HNMR spectra of the synthesized compounds in deuteriated DMSO were recorded on BRUKER AVANCE II $400 \mathrm{MHz}$ NMR Spectrometer instrument using TMS as the internal standard. Mass spectra were recorded using LC-MSD-TranpSL2010A SHIMADZU using Dimethyl-sulphoxide (DMSO) as a solvent. TLC was performed using silica gel GF254 coated plates of $0.25 \mathrm{~mm}$ thickness. Ethyl acetate, petroleum ether, chloroform (0.6:0.8:8.6) were used as a solvent and iodine vapors as a visualizing agent.

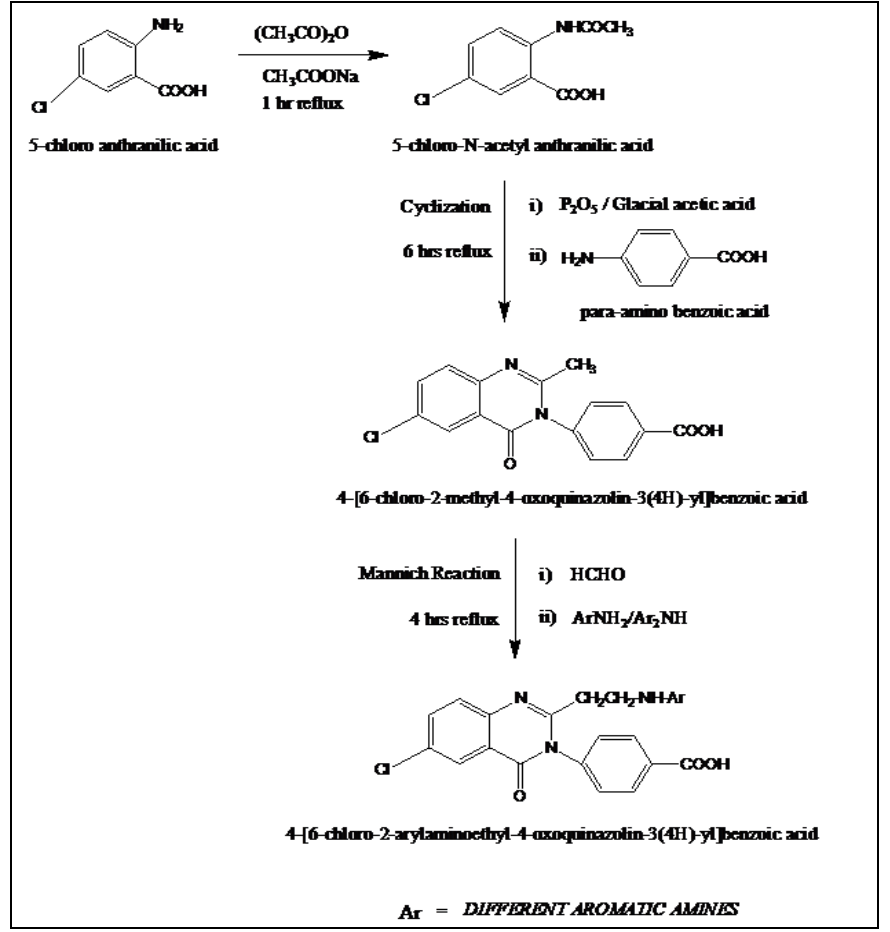

SCHEME OF SYNTHESIS

TABLE 1: LIST OF VARIOUS AROMATIC AMINES

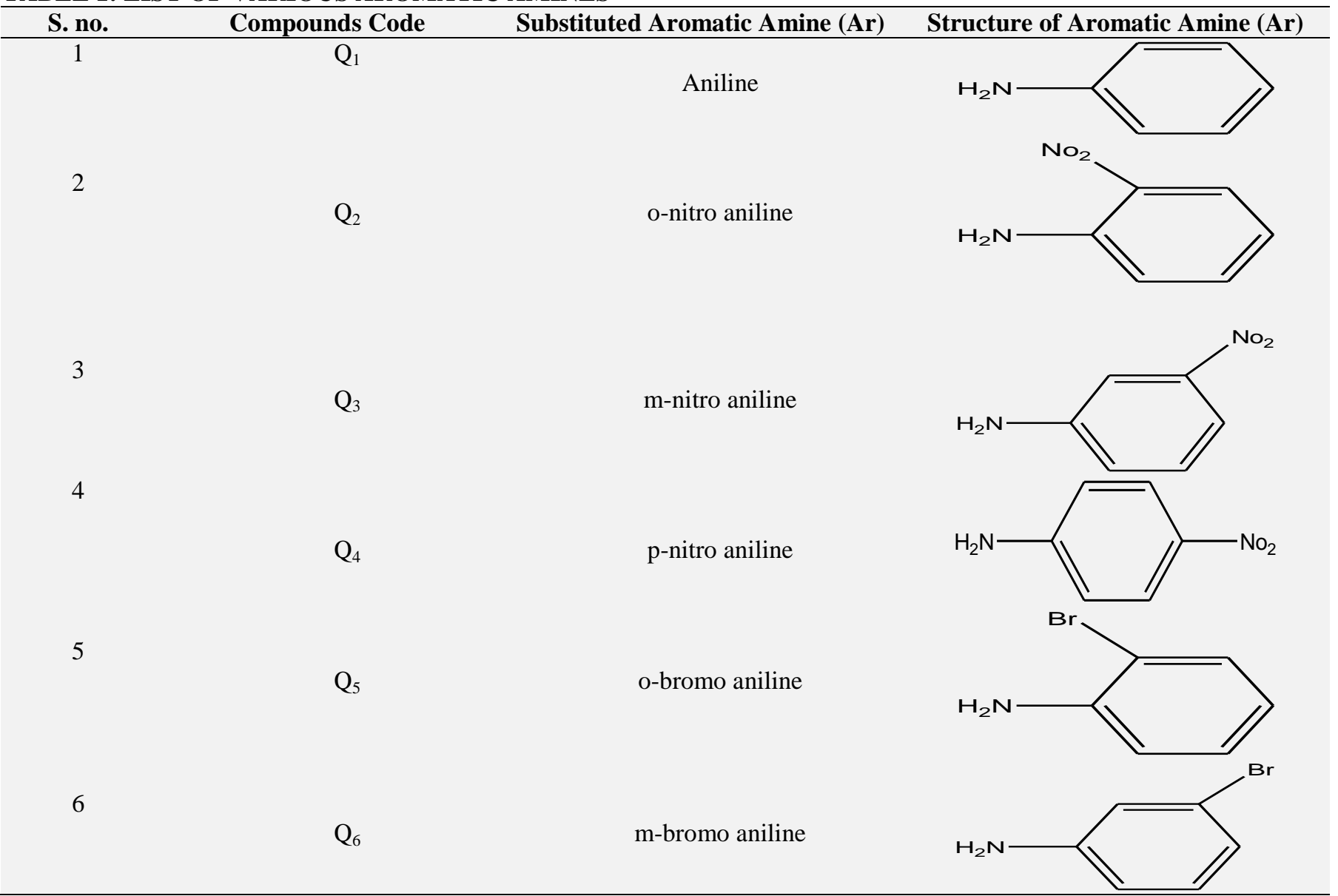




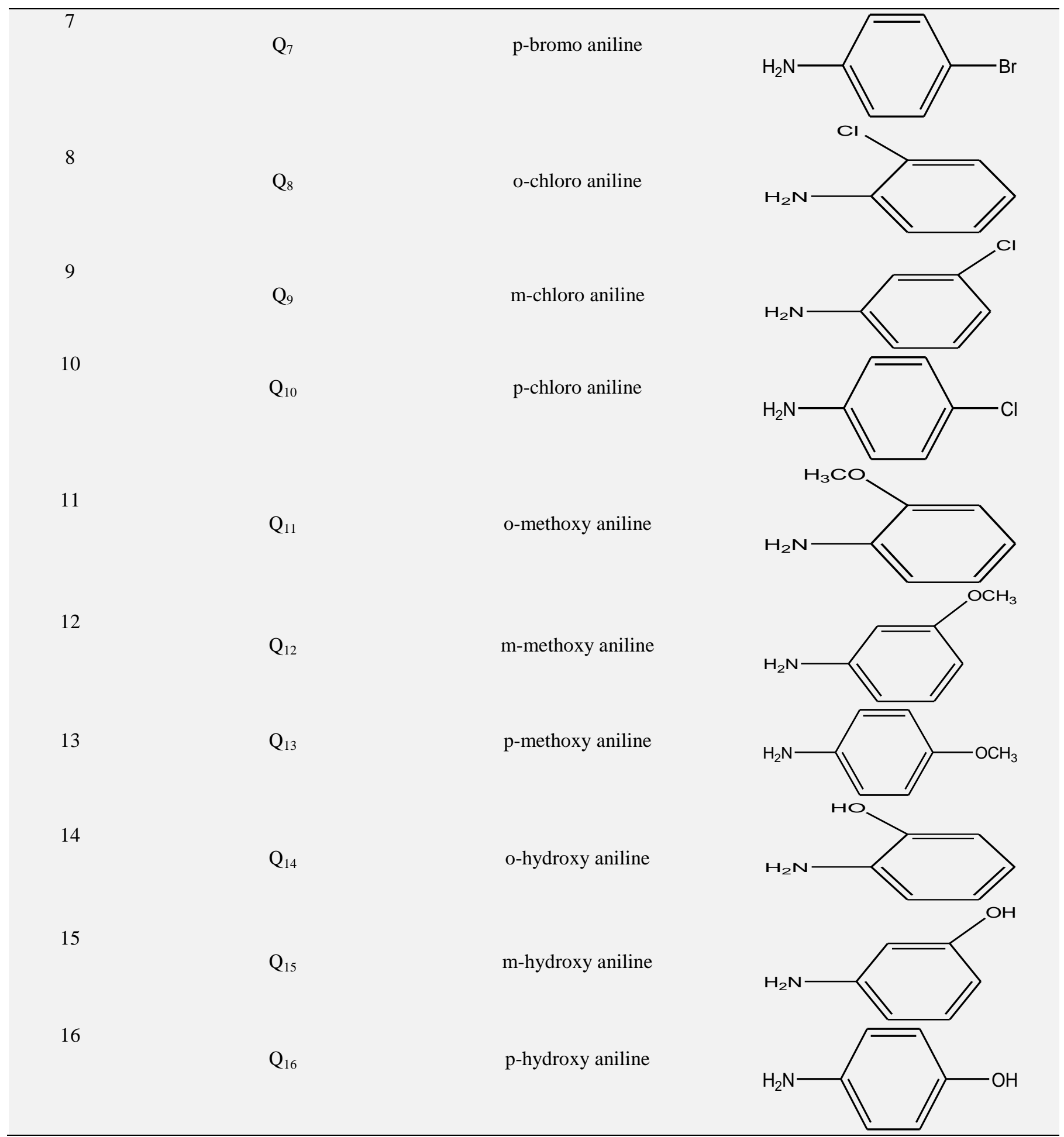

The Experimental Work Comprises in Three Steps:

Step-I: Synthesis of 5-chloro-N-acetyl anthranilic acid from 5-chloro anthranilic acid.

Step-II: Synthesis of 4-[6-chloro-2-methyl-4-oxo quinazolin-3(4H)-yl] benzoic acid.

Step-III: Synthesis of various derivatives of quinazolinone by mannich reaction.
Step-I: General Procedure for the Synthesis of 5-Chloro-N-Acetyl Anthranilic Acid From 5Chloro Anthranilic Acid (Intermediate-I): 5Chloro anthranilic acid (0.02 moles) was mixed with equimolar quantities of anhydrous sodium acetate $(0.03$ moles $)$ and acetic anhydride (0.04 moles in slight excess) and refluxed on sand bath under the anhydrous condition for $1 \mathrm{~h}$. Then the reaction mixture was poured into ice-cold water, 
and the crude product was filtered and dried. The dried crude product was recrystallized from ethanol. Yield: $81.34 \%$ M.P.: $188-190^{\circ} \mathrm{C}$.

Step-II: General Procedure for the Synthesis of 4-[6-Chloro-2-Methyl-4-Oxo Quinazolin- 3(4H)YI] Benzoic Acid (Intermediate-II): 5-Chloro-Nacetyl anthranilic acid (0.01 moles) was added to a mixture of 4-Amino benzoic acid (0.02 moles), Phosphorus pentoxide (0.03 moles) and Glacial acetic acid $(15 \mathrm{ml})$ and the mixture were refluxed under the anhydrous condition for $6 \mathrm{~h}$. Then the reaction mixture was poured into $10 \%$ Sodium bicarbonate solution $(50 \mathrm{ml})$, and the crude product was filtered and dried. The dried crude product was recrystallized from ethanol. Yield: $76.67 \%$ M.P.: 220-222 ${ }^{\circ} \mathrm{C}$.

Step-III: General Procedure for the Synthesis of Various Derivatives of Quinazolinone By Mannich Reaction (Q1-Q16) 4-[6-Chloro-2Arylaminoethyl-4-Oxoquinazolin-3(4H)-Yl]

Benzoic Acid: A mixture of 4-[6-Chloro-2-methyl4-oxoquinazolin-3(4H)-yl] benzoic acid (0.01 mole), various aromatic amines (0.02 mole) and formaldehyde $(0.02$ mole) were taken in methanol $(80 \mathrm{ml})$, and the reaction mixture was refluxed for 4 $\mathrm{h}$. The completion of the reaction was monitored by TLC. The excess of the solvent was distilled off, and the residue was recrystallized from acetone to give the final product.

Q1: 4-(6-Chloro -4-Oxo- 2-(2-(Phenylamino) Ethyl) Quinazolin-3 (4H)-Yl) Benzoic Acid: Dark brown colored solid, Molecular formula: $\mathrm{C}_{23} \mathrm{H}_{18} \mathrm{ClN}_{3} \mathrm{O}_{3}$, Molecular weight: 419.86, Yield: 69.22\%, M.P.: $176-178{ }^{\circ} \mathrm{C}, \mathrm{R}_{\mathrm{f}}$ value: 0.79 , FT-IR $\left(\mathrm{KBr}, \mathrm{cm}^{-1}\right)$ : 3407.07 (N-H Str.), 2905.12 (C-H Str.), 1609.91 (C=C Str.), 1711.94 (C=O Str.), 1250.04 (C-N Str.), 734.56 (Ar C-H Bend.).

${ }^{1} \mathrm{H}-\mathrm{NMR}$ (400 MHz, DMSO, $\delta$ ppm): 1.55 (t, 2H, $\left.\mathrm{CH}_{2}\right), 3.22\left(\mathrm{q}, 2 \mathrm{H}, \mathrm{CH}_{2}\right), 4.13\left(\mathrm{t},{ }^{1} \mathrm{H}, \mathrm{NH}\right), 6.38$ $8.10(\mathrm{~m}, 12 \mathrm{H}, \mathrm{Ar} \mathrm{H}), 11.10\left(\mathrm{~s},{ }^{1} \mathrm{H}, \mathrm{COOH}\right)$. Mass Spectra: m/z: $421.67\left(\mathrm{M}^{+2}\right)$. Elemental Analysis, \% found (\% required): C 65.64 (65.79); H 4.28 (4.32); N 9.93 (10.01); O 11.32 (11.43); Cl 8.38 (8.44).

Q2: 4-(6-Chloro -2-(2-(2- Nitrophenylamino) Ethyl)-4-Oxoquinazolin-3(4H)-Yl) Benzoic Acid: Yellowish brown colored solid, Molecular formula: $\mathrm{C}_{23} \mathrm{H}_{17} \mathrm{C}_{1} \mathrm{~N}_{4} \mathrm{O}_{5}$, Molecular weight: 464.86, Yield:
68.32\%, M.P.: $152-154{ }^{\circ} \mathrm{C}, \mathrm{R}_{\mathrm{f}}$ value: 0.79 , FT-IR $\left(\mathrm{KBr}, \mathrm{cm}^{-1}\right)$ : 3434.67 (N-H Str.), 2991.28 (C-H Str.), 1629.41 (C=C Str.), 1701.03 (C=O Str.), 1254.04 (C-N Str.), 743.35 (Ar C-H Bend.), 1497.09 (Ar N=O Str.). ${ }^{1} \mathrm{H}-\mathrm{NMR}(400 \mathrm{MHz}$, DMSO, $\delta \mathrm{ppm}): 1.57\left(\mathrm{t}, 2 \mathrm{H}, \mathrm{CH}_{2}\right), 3.31$ (q, 2H, $\left.\mathrm{CH}_{2}\right), 4.20\left(\mathrm{t},{ }^{1} \mathrm{H}, \mathrm{NH}\right), 6.70-8.14\left(\mathrm{~m},{ }^{11} \mathrm{H}\right.$, Ar H$)$, 11.12 (s, $\left.{ }^{1} \mathrm{H}, \mathrm{COOH}\right)$. Mass Spectra: m/z: 466.43 $\left(\mathrm{M}^{+2}\right)$. Elemental Analysis, \% found (\% required): C 59.34 (59.43); H 3.65 (3.69); N 11.96 (12.05); O 17.15 (17.21); Cl 7.55 (7.63).

Q3: 4-(6- Chloro-2-(2-(3- Nitrophenylamino) Ethyl)-4-Oxoquinazolin-3(4H)-Yl) Benzoic Acid: Creamish yellow colored solid, Molecular formula: $\mathrm{C}_{23} \mathrm{H}_{17} \mathrm{C}_{1} \mathrm{~N}_{4} \mathrm{O}_{5}$, Molecular weight: 464.86, Yield: 67.22\%, M.P.: $168-170{ }^{\circ} \mathrm{C}, \mathrm{R}_{\mathrm{f}}$ value: 0.80 , FT-IR $\left(\mathrm{KBr}, \mathrm{cm}^{-1}\right)$ : 3396.21 (N-H Str.), 2895.47 (C-H Str.), 1599.85 (C=C Str.), 1704.19 (C=O Str.), 1222.75 (C-N Str.), 742.59 (Ar C-H Bend.), 1452.39 (Ar N=O Str.). 1H-NMR (400 MHz, DMSO, $\delta$ ppm): $1.58\left(\mathrm{t}, 2 \mathrm{H}, \mathrm{CH}_{2}\right), 3.29$ (q, 2H, $\left.\mathrm{CH}_{2}\right), 4.17(\mathrm{t}, 1 \mathrm{H}, \mathrm{NH}), 6.75-8.12(\mathrm{~m}, 11 \mathrm{H}, \mathrm{Ar} \mathrm{H})$, 11.00 (s, ${ }^{1} \mathrm{H}, \mathrm{COOH}$ ). Mass Spectra: m/z: 466.57 $\left(\mathrm{M}^{+2}\right)$. Elemental Analysis, \% found (\% required): C 59.35 (59.43); H 3.51 (3.69); N 12.08 (12.05); O 17.12 (17.21); Cl 7.52 (7.63).

Q4: 4-(6-Chloro -2-(2-(4- Nitrophenylamino) Ethyl)-4-Oxoquinazolin-3(4H)-Yl) Benzoic Acid: Pale yellow colored solid, Molecular formula: $\mathrm{C}_{23} \mathrm{H}_{17} \mathrm{C}_{1} \mathrm{~N}_{4} \mathrm{O}_{5}$, Molecular weight: 464.86, Yield: 67.77\%, M.P.: $178-180{ }^{\circ} \mathrm{C}, \mathrm{R}_{\mathrm{f}}$ value: 0.76 , FT-IR $\left(\mathrm{KBr}, \mathrm{cm}^{-1}\right)$ : 3434.65 (N-H Str.), 2917.59 (C-H Str.), 1657.18 (C=C Str.), 1754.78 (C=O Str.), 1259.94 (C-N Str.), 796.20 (Ar C-H Bend.), 1470.73 (Ar N=O Str.). 1H-NMR (400 MHz, DMSO, $\delta$ ppm): $1.61\left(\mathrm{t}, 2 \mathrm{H}, \mathrm{CH}_{2}\right), 3.15(\mathrm{q}, 2 \mathrm{H}$, $\left.\mathrm{CH}_{2}\right), 4.11(\mathrm{t}, 1 \mathrm{H}, \mathrm{NH}), 6.65-8.12(\mathrm{~m}, 11 \mathrm{H}, \mathrm{Ar} \mathrm{H})$, 11.15 (s, 1H, COOH). Elemental Analysis, \% found (\% required): C 59.37 (59.43); H 3.61 (3.69); N 11.95 (12.05); O 17.13 (17.21); Cl 7.60 (7.63).

Q5: 4-(2-(2-(2- Bromophenylamino) Ethyl)-6Chloro-4- Oxoquinazolin-3(4h)-Yl) Benzoic Acid: Pale red colored solid, Molecular formula: $\mathrm{C}_{23} \mathrm{H}_{17} \mathrm{BrC}_{1} \mathrm{~N}_{3} \mathrm{O}_{3}$, Molecular weight: 498.76, Yield: 73.24\%, M.P.: $155-157^{\circ} \mathrm{C}, \mathrm{R}_{\mathrm{f}}$ value: 0.76 , FT-IR $\left(\mathrm{KBr}, \mathrm{cm}^{-1}\right)$ : 3363.76 (N-H Str.), 2898.37 (C-H Str.), 1599.58 (C=C Str.), 1679.43 (C=O Str.), 1258.02 (C-N Str.), 768.37 (Ar C-H Bend.), 678.46 
(Ar C-Br Bend.). 1H-NMR (400 MHz, DMSO, $\delta$ ppm): 1.63 (t, 2H, CH2), 3.12 (q, 2H, CH2), 4.14 $(\mathrm{t}, 1 \mathrm{H}, \mathrm{NH}), 6.34-8.11(\mathrm{~m}, 11 \mathrm{H}, \operatorname{Ar} \mathrm{H}), 11.10(\mathrm{~s}$, 1H, COOH). Mass Spectra: $\mathrm{m} / \mathrm{z}$ : $500.07\left(\mathrm{M}^{+2}\right)$. Elemental Analysis, \% found (\% required): C 55.32 (55.39); H 3.40 (3.44); N 8.35 (8.42); O 9.57 (9.62); Cl 7.07 (7.11); Br 15.99 (16.02).

Q6: 4-(2-(2-(3-Bromophenylamino) Ethyl) -6Chloro-4- Oxoquinazolin-3 (4H)-Yl) Benzoic Acid: Light red colored solid, Molecular formula: $\mathrm{C}_{23} \mathrm{H}_{17} \mathrm{BrC}_{1} \mathrm{~N}_{3} \mathrm{O}_{3}$, Molecular weight: 498.76, Yield: 72.84\%, M.P.: $158-160{ }^{\circ} \mathrm{C}, \mathrm{R}_{\mathrm{f}}$ value: 0.74 , FT-IR $\left(\mathrm{KBr}, \mathrm{cm}^{-1}\right)$ : 3320.88 (N-H Str.), 2809.58 (C-H Str.), 1589.43 (C=C Str.), 1666.88 (C=O Str.), 1258.46 (C-N Str.), 718.27 (Ar C-H Bend.), 650.43 (Ar C-Br Bend.). 1H-NMR (400 MHz, DMSO, $\delta$ ppm): 1.64 (t, 2H, $\mathrm{CH}_{2}$ ), 3.10 (q, 2H, $\mathrm{CH}_{2}$ ), 4.16 (t, $1 \mathrm{H}, \mathrm{NH}), 6.37-8.13(\mathrm{~m}, 11 \mathrm{H}, \mathrm{Ar} \mathrm{H}), 11.08$ (s, 1H, $\mathrm{COOH})$. Mass Spectra: $\mathrm{m} / \mathrm{z}$ : $500.04 \quad\left(\mathrm{M}^{+2}\right)$. Elemental Analysis, \% found (\% required): C 55.32 (55.39); H 3.38 (3.44); N 8.36 (8.42); O 9.57 (9.62); Cl 7.10 (7.11); Br 15.98 (16.02).

Q7: 4-(2-(2-(4-Bromophenylamino) Ethyl) -6Chloro-4- Oxoquinazolin-3 (4H)-Yl) Benzoic Acid: Greyish red colored solid, Molecular formula: $\mathrm{C}_{23} \mathrm{H}_{1} 7 \mathrm{BrClN}_{3} \mathrm{O}_{3}$, Molecular weight: 498.76, Yield: $70.24 \%$, M.P.: $160-162^{\circ} \mathrm{C}, \mathrm{R}_{\mathrm{f}}$ value: 0.70, FT-IR (KBr, cm $\left.{ }^{-1}\right): 3334.67$ (N-H Str.), 2849.31 (C-H Str.), 1597.36(C=C Str.), 1693.03 (C=O Str.), 1255.20 (C-N Str.), 717.26 (Ar C-H Bend.), 637.77 (Ar C-Br Bend.). 1H-NMR (400 $\mathrm{MHz}, \mathrm{DMSO}, \delta \mathrm{ppm}): 1.57$ (t, 2H, $\left.\mathrm{CH}_{2}\right), 3.14$ (q, $\left.2 \mathrm{H}, \mathrm{CH}_{2}\right), 3.94$ (t, 1H, NH), 6.29-8.10 (m, 11H, Ar $\mathrm{H}), 11.05$ (s, 1H, COOH). Elemental Analysis, \% found (\% required): C 55.36 (55.39); H 3.41 (3.44); N 8.34 (8.42); O 9.59 (9.62); Cl 7.02 (7.11); Br 15.94 (16.02).

Q8: 4-(6-Chloro-2-(2-(2- Chlorophenylamino) Ethyl)-4- Oxoquinazolin-3(4H)-Yl) Benzoic Acid: Dark Brown colored solid, Molecular formula: $\mathrm{C}_{23} \mathrm{H}_{17} \mathrm{C}_{12} \mathrm{~N}_{3} \mathrm{O}_{3}$, Molecular weight: 454.31, Yield: $69.30 \%$, M.P.: $204-206^{\circ} \mathrm{C}, \mathrm{R}_{\mathrm{f}}$ value: 0.71, FT-IR (KBr, cm $\left.{ }^{-1}\right): 3371.64$ (N-H Str.), 2863.85 (C-H Str.), 1572.74(C=C Str.), 1711.10 (C=O Str.), 1259.33 (C-N Str.), 713.32 (Ar C-H Bend.), 654.23 (Ar C-Cl Bend.). 1H-NMR (400 $\mathrm{MHz}, \mathrm{DMSO}, \delta \mathrm{ppm}$ ): 1.59 (t, 2H, CH2), 2.98 (q, 2H, CH2), 4.03 (t, 1H, NH), 6.37-8.12 (m, 11H, Ar
H), $11.02(\mathrm{~s}, 1 \mathrm{H}, \mathrm{COOH})$. Mass Spectra: m/z: $456.39\left(\mathrm{M}^{+2}\right)$. Elemental Analysis, \% found $(\%$ required): C 60.77 (60.81); H 3.72 (3.77); N 9.20 (9.25); O 10.51 (10.57); Cl 15.58 (15.61).

Q9: 4-(6-Chloro-2-(2-(3- Chlorophenylamino) Ethyl)- 4-Oxoquinazolin-3 (4H)-Yl) Benzoic Acid: Pale Brown colored solid, Molecular formula: $\mathrm{C}_{23} \mathrm{H}_{17} \mathrm{C}_{12} \mathrm{~N}_{3} \mathrm{O}_{3}$, Molecular weight: 454.31, Yield: $67.84 \%$, M.P.: $210-212^{\circ} \mathrm{C}, \mathrm{R}_{\mathrm{f}}$ value: 0.78, FT-IR (KBr, cm $\left.{ }^{-1}\right): 3394.98$ (N-H Str.), 2858.37 (C-H Str.), 1504.84 (C=C Str.), 1724.98 (C=O Str.), 1209.33 (C-N Str.), 710.11 (Ar C-H Bend.), 673.29 (Ar C-Cl Bend.). 1H-NMR (400 $\mathrm{MHz}, \mathrm{DMSO}, \delta \mathrm{ppm}): 1.62\left(\mathrm{t}, 2 \mathrm{H}, \mathrm{CH}_{2}\right), 3.11(\mathrm{q}$, $\left.2 \mathrm{H}, \mathrm{CH}_{2}\right), 4.08(\mathrm{t}, 1 \mathrm{H}, \mathrm{NH}), 6.30-8.07(\mathrm{~m}, 11 \mathrm{H}, \mathrm{Ar}$ $\mathrm{H}), 11.01(\mathrm{~s}, 1 \mathrm{H}, \mathrm{COOH})$. Elemental Analysis, \% found (\% required): C 60.77 (60.81); H 3.71 (3.77); N 9.22 (9.25); O 10.54 (10.57); Cl 15.58 (15.61).

\section{Q10: 4-(6-Chloro-2-(2-(4-Chlorophenylamino)} Ethyl)-4- Oxoquinazolin- 3(4H)-Yl) Benzoic Acid: Creamish Brown colored solid, Molecular formula: $\mathrm{C}_{23} \mathrm{H}_{17} \mathrm{C}_{12} \mathrm{~N}_{3} \mathrm{O}_{3}$, Molecular weight: 454.31, Yield: $66.67 \%$, M.P.: $209-211^{\circ} \mathrm{C}, \mathrm{R}_{\mathrm{f}}$ value: 0.69, FT-IR (KBr, cm $\left.{ }^{-1}\right): 3375.68$ (N-H Str.), 2719.68 (C-H Str.), 1531.28 (C=C Str.), 1717.59 (C=O Str.), 1207.04 (C-N Str.), 761.10 (Ar C-H Bend.), 640.39 (Ar C-Cl Bend.). 1H-NMR (400 $\mathrm{MHz}, \mathrm{DMSO}, \delta \mathrm{ppm}): 1.60\left(\mathrm{t}, 2 \mathrm{H}, \mathrm{CH}_{2}\right), 3.17$ (q, $\left.2 \mathrm{H}, \mathrm{CH}_{2}\right), 4.10(\mathrm{t}, 1 \mathrm{H}, \mathrm{NH}), 6.35-8.11(\mathrm{~m}, 11 \mathrm{H}, \mathrm{Ar}$ $\mathrm{H}), 10.89(\mathrm{~s}, 1 \mathrm{H}, \mathrm{COOH})$. Mass Spectra: $\mathrm{m} / \mathrm{z}$ : $456.69\left(\mathrm{M}^{+2}\right)$. Elemental Analysis, \% found $(\%$ required): C 60.78 (60.81); H 3.74 (3.77); N 9.19 (9.25); O 10.50 (10.57); Cl 15.58 (15.61).

\section{Q11: 4-(6-Chloro-2-(2-(2-Methoxyphenylamino)} Ethyl)-4- Oxoquinazolin-3(4H)-Yl) Benzoic Acid: Yellowish White colored solid, Molecular formula: $\mathrm{C}_{24} \mathrm{H}_{20} \mathrm{C}_{1} \mathrm{~N}_{3} \mathrm{O}_{4}$, Molecular weight: 449.89, Yield: $64.54 \%$, M.P.: $147-149^{\circ} \mathrm{C}, \mathrm{R}_{\mathrm{f}}$ value: 0.65 , FT-IR (KBr, cm $\left.{ }^{-1}\right)$ : 3369.95 (N-H Str.), 2809.33 (C-H Str.), 1517.09(C=C Str.), 1694.15 (C=O Str.), 1217.93 (C-N Str.), 710.77 (Ar C-H Bend.). 1HNMR (400 MHz, DMSO, $\delta$ ppm): 1.61 (t, 2H, $\mathrm{CH}_{2}$ ), 3.22 (q, 2H, $\left.\mathrm{CH}_{2}\right), 4.16(\mathrm{t}, 1 \mathrm{H}, \mathrm{NH}), 3.71$ (s, 3H, OCH3), 6.31-8.09 (m, 11H, Ar H), 11.04 (s, $1 \mathrm{H}, \mathrm{COOH})$. Mass Spectra: m/z: $451.27\left(\mathrm{M}^{+2}\right)$. Elemental Analysis, \% found (\% required): C 63.98 (64.07); H 4.45 (4.48); N 9.30 (9.34); O 14.19 (14.23); Cl 7.84 (7.88). 
Q12: 4-(6-Chloro-2-(2-(3-Methoxyphenylamino) Ethyl)-4- Oxoquinazolin-3(4H)-Yl) Benzoic Acid: Creamish White colored solid, Molecular formula: $\mathrm{C}^{24} \mathrm{H}^{20} \mathrm{C}^{1} \mathrm{~N}^{3} \mathrm{O}^{4}$, Molecular weight: 449.89 , Yield: $60.53 \%$, M.P.: $152-154^{\circ} \mathrm{C}, \mathrm{R}_{\mathrm{f}}$ value: 0.68 , FT-IR (KBr, cm ${ }^{-1}$ ): 3396.75 (N-H Str.), 2898.47 (C-H Str.), 1531.07(C=C Str.), 1704.58 (C=O Str.), 1239.75 (C-N Str.), 719.43 (Ar C-H Bend.). 1HNMR (400 MHz, DMSO, $\delta$ ppm): 1.58 (t, 2H, $\left.\mathrm{CH}_{2}\right), 3.11$ (q, 2H, CH2), 4.13 (t, 1H, NH), 3.75 (s, $\left.3 \mathrm{H}, \mathrm{OCH}_{3}\right), 5.91-8.0(\mathrm{~m}, 11 \mathrm{H}, \mathrm{Ar} \mathrm{H}), 11.01(\mathrm{~s}, 1 \mathrm{H}$, $\mathrm{COOH})$. Elemental Analysis, \% found (\% required): C 63.99 (64.07); H 4.45 (4.48); N 9.32(9.34); O 14.19 (14.23); Cl 7.81 (7.88).

Q13: 4-(6-Chloro-2-(2-(4-Methoxyphenylamino) Ethyl)-4 -Oxoquinazolin-3(4H)-Yl) Benzoic Acid: White Brown colored solid, Molecular formula: $\mathrm{C}_{24} \mathrm{H}_{20} \mathrm{C}_{1} \mathrm{~N}_{3} \mathrm{O}_{4}$, Molecular weight: 449.89, Yield: $70.79 \%$, M.P.: $138-140{ }^{\circ} \mathrm{C}, \mathrm{R}_{\mathrm{f}}$ value: 0.66 , FT-IR (KBr, cm ${ }^{-1}$ ): 3421.07 (N-H Str.), 2918.12 (C-H Str.), 1546.13(C=C Str.), 1719.41 (C=O Str.), 1208.08 (C-N Str.), 735.04 (Ar C-H Bend.). 1HNMR (400 MHz, DMSO, $\delta$ ppm): $1.64(\mathrm{t}, 2 \mathrm{H}$, $\left.\mathrm{CH}_{2}\right), 3.14$ (q, 2H, $\left.\mathrm{CH}_{2}\right), 4.07(\mathrm{t}, 1 \mathrm{H}, \mathrm{NH}), 3.72$ (s, $\left.3 \mathrm{H}, \mathrm{OCH}_{3}\right), 6.30-8.03(\mathrm{~m}, 11 \mathrm{H}, \mathrm{Ar} \mathrm{H}), 10.86(\mathrm{~s}$, 1H, COOH). Mass Spectra: m/z: $451.35\left(\mathrm{M}^{+2}\right)$. Elemental Analysis, \% found (\% required):C 64.01 (64.07); H 4.46 (4.48); N 9.30(9.34); O 14.18 (14.23); Cl 7.80 (7.88).

Q14: 4-(6-Chloro-2-(2-(2-Hydroxyphenylamino) Ethyl)-4 -Oxoquinazolin-3(4H)-Yl) Benzoic Acid: Greyish Black colored solid, Molecular formula: $\mathrm{C}_{23} \mathrm{H}_{18} \mathrm{C}_{1} \mathrm{~N}_{3} \mathrm{O}_{4}$, Molecular weight: 435.86, Yield: $72.11 \%$, M.P.: $133-135^{\circ} \mathrm{C}, \mathrm{R}_{\mathrm{f}}$ value: 0.71 , FT-IR (KBr, cm-1): 3478.22 (N-H Str.), 2934.89 (C-H Str.), 1530.86(C=C Str.), 1643.50 (C=O Str.), 1209.79 (C-N Str.), 737.35 (Ar C-H Bend.), 3446.18 (Ar C-OH Str.). 1H-NMR (400 MHz, DMSO, $\delta$ ppm): 1.69 (t, 2H, $\left.\mathrm{CH}_{2}\right), 3.25$ (q, 2H, $\left.\mathrm{CH}_{2}\right), 4.01(\mathrm{t}, 1 \mathrm{H}, \mathrm{NH}), 5.10(\mathrm{~s}, 1 \mathrm{H}, \mathrm{OH}), 6.24-8.12$ $(\mathrm{m}, 11 \mathrm{H}, \operatorname{Ar} \mathrm{H}), 11.12(\mathrm{~s}, 1 \mathrm{H}, \mathrm{COOH})$. Mass Spectra: m/z: $437.11(\mathrm{M}+2)$. Elemental Analysis, \% found (\% required): C 63.35 (63.38); H 4.10 (4.16); N 9.59(9.64); O 14.66 (14.68); Cl 8.09 (8.13).

Q15: 4-(6-Chloro-2-(2-(3-Hydroxyphenylamino) Ethyl)- 4-Oxoquinazolin-3(4H)-Yl) Benzoic Acid: Yellowish Black colored solid, Molecular formula: $\mathrm{C}_{23} \mathrm{H}_{18} \mathrm{C}_{1} \mathrm{~N}_{3} \mathrm{O}_{4}$, Molecular weight: 435.86, Yield: $74.38 \%$, M.P.: $138-140^{\circ} \mathrm{C}, \mathrm{R}_{\mathrm{f}}$ value: 0.74 ,
FT-IR (KBr, cm $\left.{ }^{-1}\right): 3477.38$ (N-H Str.), 2979.13 (C-H Str.), 1531.84(C=C Str.), 1622.98 (C=O Str.), 1207.57 (C-N Str.), 762.11 (Ar C-H Bend.), 3446.93 (Ar C-OH Str.). 1H-NMR (400 MHz, DMSO, $\delta$ ppm): $1.65\left(\mathrm{t}, 2 \mathrm{H}, \mathrm{CH}_{2}\right), 3.19(\mathrm{q}, 2 \mathrm{H}$, $\left.\mathrm{CH}_{2}\right), 4.04(\mathrm{t}, 1 \mathrm{H}, \mathrm{NH}), 5.07$ (s, 1H, OH), 5.89-8.14 (m, 11H, Ar H), 11.03 (s, 1H, COOH). Elemental Analysis, \% found (\% required): C 63.33 (63.38); H 4.11 (4.16); N 9.60 (9.64); O 14.63 (14.68); Cl $8.08(8.13)$.

Q16: 4-(6-Chloro-2-(2-(4-Hydroxyphenylamino) Ethyl)- 4-Oxoquinazolin-3(4H)-Yl) Benzoic Acid: Black Red colored solid, Molecular formula: $\mathrm{C}_{23} \mathrm{H}_{18} \mathrm{C}_{1} \mathrm{~N}_{3} \mathrm{O}_{4}$, Molecular weight: 435.86, Yield: 70.25\%, M.P.: 144-146oC, $\mathrm{R}_{\mathrm{f}}$ value: 0.69, FT-IR $\left(\mathrm{KBr}, \mathrm{cm}^{-1}\right)$ : 3377.38 (N-H Str.), 2979.49 (C-H Str.), 1572.38(C=C Str.), 1617.87 (C=O Str.), 1249.85 (C-N Str.), 733.59 (Ar C-H Bend.), 3315.87 (Ar C-OH Str.). 1H-NMR (400 MHz, DMSO, $\delta \mathrm{ppm}): 1.63$ (t, 2H, $\left.\mathrm{CH}_{2}\right), 3.18(\mathrm{q}, 2 \mathrm{H}$, $\left.\mathrm{CH}_{2}\right), 4.11(\mathrm{t}, 1 \mathrm{H}, \mathrm{NH}), 5.02(\mathrm{~s}, 1 \mathrm{H}, \mathrm{OH}), 6.25-8.11$ $(\mathrm{m}, 11 \mathrm{H}, \mathrm{Ar} \mathrm{H}), 11.10$ (s, 1H, COOH). Elemental Analysis, \% found (\% required): C 63.31 (63.38); H 4.11 (4.16); N 9.60 (9.64); O 14.66 (14.68); Cl 8.07 (8.13).

\section{Biological Study:}

Evaluation of Anti- Inflammatory Activity 15, 16: Adult albino rats of both sexes weighing between 120 and $150 \mathrm{~g}$ were used. Rats were uniformly hydrated by giving $3 \mathrm{ml}$ water/rat through gastric inoculation to reduce variability to oedema response. Animals were divided into 18 groups, each of five animals. The control group was given saline solution containing few drops of Tween 80 . Diclofenac sodium $(50 \mathrm{mg} / \mathrm{kg})$ was taken as a standard drug for comparison, and compounds under examination $(100 \mathrm{mg} / \mathrm{kg})$ were suspended in distilled water with the aid of few drops of Tween 80 and were given orally $1 \mathrm{~h}$ before induction of inflammation. Induction of inflammation was performed by S.C. injection of $50 \mu \mathrm{l}$ of $1 \%$ carrageenan-sodium gel into the sub-plantar region of the right hind paw. The dorsoventral diameter (thickness) of the right and left hind paw of each rat was measured using a pair of dial thickness gauge callipers accurate to $0.001 \mathrm{~cm} 0.5,1,2$, and $3 \mathrm{hr}$ after induction of inflammation. The left hind paw diameter served as a control for the degree of inflammation in the right hind paw. The percentage 
of anti-inflammatory activity (\% inhibition of inflammation) was calculated according to the following equation:

$$
\% \text { inhibition }=(\mathrm{Wc}-\mathrm{Wt} / \mathrm{Wc}) \times 100
$$

$\mathrm{Wt}$ : is the mean increase in paw thickness in rats treated with the tested compounds, Wc: is the mean increase in paw thickness in the control group.

\section{Biochemical Estimation:}

Estimation of Cytokines Tumor Necrosis Factor Alpha, Interleukin 6 and Interleukin 1 In Serum: After completion of the carrageenaninduced paw edema experiment, the rats were anesthetized, and blood samples were collected from the orbital sinus. The serum was separated by allowing blood to clot followed by centrifugation, and was stored at $20^{\circ} \mathrm{C}$ until use. TNF- $\alpha$, IL-6, IL1 from each sample were measured in duplicate with highly sensitive rat TNF- $\alpha$ Elisa Kit (Bio molecular Integrations), rat IL-6 Elisa Kit (Koma Biotech), rat IL-1 Elisa Kit (Boster Biological Technology Ltd) respectively, specifically designed for rats, according to manufacturer's instructions.

\section{Determination of Tissue Lipid Peroxidation:}

MDA Assay: The Malonylaldehyde from carrageenan-induced edema foot was evaluated by using the thiobarbituric acid reacting substance method. Briefly, the reaction mixture containing 1 $\mathrm{ml}$ 0.67\% Thio Barbituric Acid (TBA), $1 \mathrm{ml} 20 \%$ Tri Carboxylic Acid (TCA) and $100 \mathrm{ml}$ serum were incubated at $100{ }^{\circ} \mathrm{C}$ for $30 \mathrm{~min}$, at this temperature, MDA reacted with thiobarbituric acid at acidic $\mathrm{pH}$ resulting in the formation of a red complex TBARS and centrifuged at $5000 \mathrm{rpm}$ for $15 \mathrm{~min}$. The absorbance of TBARS was determined at $532 \mathrm{~nm}$.

\section{RESULTS AND DISCUSSION:}

Chemistry: All the novel quinazolinone derivatives were synthesized, purified, and separated by using column chromatography or recrystallization method. Synthesized compounds were characterized by using elemental analysis, FT-IR, ${ }^{1} \mathrm{HNMR}$, and Mass Spectrometric studies. The integration curves fully support the orientation of protons in the analyzed compounds. Furthermore, all the compounds demonstrated the characteristic chemical shifts for the quinazolinone nucleus. Additionally, synthesized compounds were analyzed by mass spectra and indicated no difference in the fragmentation pattern among the set of synthesized series.

Anti- Inflammatory Activity: The antiinflammatory activity was assessed by using Carrageenan-induced rat paw edema using Diclofenac sodium as the reference drug. The antiinflammatory activity data were obtained as the thickness of edema at $0.5 \mathrm{~h}, 1 \mathrm{~h}, 2 \mathrm{~h}$, and $3 \mathrm{~h}$ intervals and expressed in \% inhibition as shown in Tables 2 and 3. Compounds Q3, Q8, and Q15 showed excellent anti-inflammatory activity at $74.46 \%, 72.34 \%$, and $68.08 \%$ inhibition respectively at $3^{\text {rd }} \mathrm{h}$, which were nearby $78.72 \%$ inhibition of the standard Diclofenac sodium used and also greater than the other quinazolinone derivatives.

TABLE 2: OEDEMA THICKNESS OF CONTROL, DICLOFENAC SODIUM AND TESTED COMPOUNDS.

\begin{tabular}{|c|c|c|c|c|}
\hline \multirow[t]{2}{*}{ Compounds } & \multicolumn{4}{|c|}{ Oedema Thickness $(\mathrm{mm}) \pm \mathrm{SEM}$} \\
\hline & $0.5 \mathrm{~h}$ & $1 \mathrm{~h}$ & $2 \mathrm{~h}$ & $3 \mathbf{h}$ \\
\hline Diclofenac Sodium & $0.160 \pm 0.008$ & $0.150 \pm 0.006$ & $0.120 \pm 0.004$ & $0.100 \pm 0.005 * * *$ \\
\hline $\mathrm{Q}_{2}$ & $0.250 \pm 0.014$ & $0.210 \pm 0.013$ & $0.190 \pm 0.015$ & $0.160 \pm 0.010 * * *$ \\
\hline $\mathrm{Q}_{3}$ & $0.200 \pm 0.007$ & $0.180 \pm 0.009$ & $0.150 \pm 0.008$ & $0.120 \pm 0.007 * * *$ \\
\hline $\mathrm{Q}_{4}$ & $0.260 \pm 0.016$ & $0.250 \pm 0.015$ & $0.230 \pm 0.011$ & $0.200 \pm 0.014 * *$ \\
\hline $\mathrm{Q}_{7}$ & $0.300 \pm 0.004$ & $0.290 \pm 0.005$ & $0.270 \pm 0.003$ & $0.260 \pm 0.004^{* *}$ \\
\hline $\mathrm{Q}_{8}$ & $0.220 \pm 0.009$ & $0.190 \pm 0.006$ & $0.170 \pm 0.008$ & $0.130 \pm 0.008 * * *$ \\
\hline $\mathrm{Q}_{9}$ & $0.240 \pm 0.010$ & $0.220 \pm 0.011$ & $0.180 \pm 0.012$ & $0.160 \pm 0.010 * * *$ \\
\hline $\mathrm{Q}_{10}$ & $0.270 \pm 0.008$ & $0.240 \pm 0.007$ & $0.220 \pm 0.009$ & $0.190 \pm 0.006^{* *}$ \\
\hline $\mathrm{Q}_{11}$ & $0.290 \pm 0.014$ & $0.270 \pm 0.012$ & $0.260 \pm 0.013$ & $0.240 \pm 0.013 * *$ \\
\hline $\mathrm{Q}_{12}$ & $0.260 \pm 0.015$ & $0.260 \pm 0.014$ & $0.240 \pm 0.015$ & $0.220 \pm 0.012 * *$ \\
\hline
\end{tabular}

Values are expressed as mean \pm SEM of five animals in each group. $* *$ Statistically significant $(\mathrm{P}<0.05) . * * *$ Statistically significant $(\mathrm{P}<0.01)$ 
TABLE 3: \% INHIBITION OF COMPOUNDS ON CARRAGEENAN INDUCED PAW RAT PAW EDEMA

\begin{tabular}{ccccc}
\hline Compounds & \multicolumn{4}{c}{ Oedema inhibition (\%) } \\
\cline { 2 - 5 } & $\mathbf{0 . 5} \mathbf{~}$ & $\mathbf{1 ~ h}$ & $\mathbf{2 ~ h}$ & $\mathbf{3 ~ h}$ \\
\hline Control & - & - & - & - \\
Diclofenac Sodium & 57.89 & 63.41 & 72.72 & $78.72^{* * *}$ \\
$\mathrm{Q}_{1}$ & 36.84 & 46.34 & 54.54 & $63.82^{* * *}$ \\
$\mathrm{Q}_{2}$ & 34.21 & 48.78 & 56.81 & $65.95^{* * *}$ \\
$\mathrm{Q}_{3}$ & 47.36 & 56.09 & 65.90 & $74.46^{* * *}$ \\
$\mathrm{Q}_{4}$ & 31.57 & 39.02 & 47.72 & $57.44^{* *}$ \\
$\mathrm{Q}_{5}$ & 23.68 & 31.70 & 36.36 & $42.55^{* *}$ \\
$\mathrm{Q}_{6}$ & 26.31 & 36.58 & 43.18 & $51.06^{* *}$ \\
$\mathrm{Q}_{7}$ & 21.05 & 29.26 & 38.63 & $44.68^{* *}$ \\
$\mathrm{Q}_{8}$ & 42.10 & 53.65 & 61.36 & $72.34^{* * *}$ \\
$\mathrm{Q}_{9}$ & 36.84 & 46.34 & 59.09 & $65.95^{* * *}$ \\
$\mathrm{Q}_{10}$ & 28.94 & 41.46 & 50 & $59.57^{* *}$ \\
$\mathrm{Q}_{11}$ & 23.68 & 34.14 & 40.90 & $48.93^{* *}$ \\
$\mathrm{Q}_{12}$ & 31.57 & 36.58 & 45.45 & $53.19^{* *}$ \\
$\mathrm{Q}_{13}$ & 18.42 & 26.82 & 34.09 & $46.80^{* *}$ \\
$\mathrm{Q}_{14}$ & 31.57 & 41.46 & 50 & $55.31^{* *}$ \\
$\mathrm{Q}_{15}$ & 39.47 & 48.78 & 59.09 & $68.08^{* * *}$ \\
$\mathrm{Q}_{16}$ & 36.84 & 43.90 & 42.27 & $61.70^{* * *}$ \\
\hline
\end{tabular}

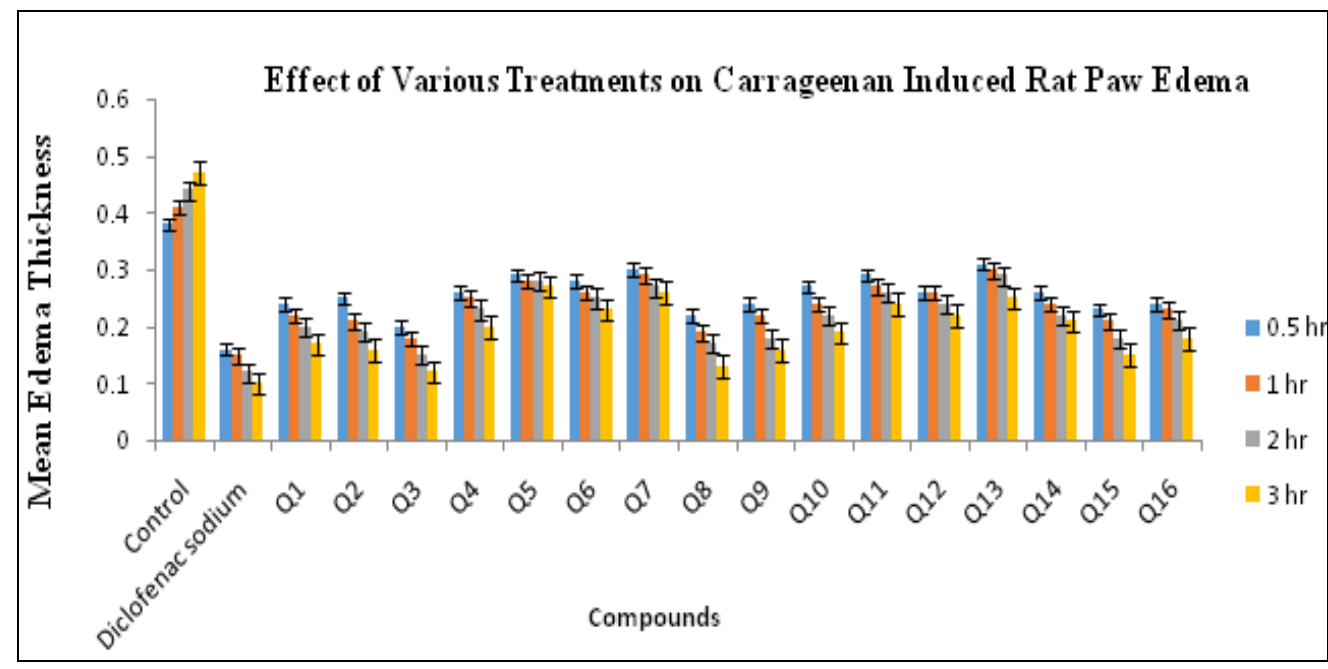

FIG. 1: EFFECT OF VARIOUS TREATMENTS ON MEAN EDEMA THICKNESS IN CARRAGEENAN INDUCED RAT PAW EDEMA

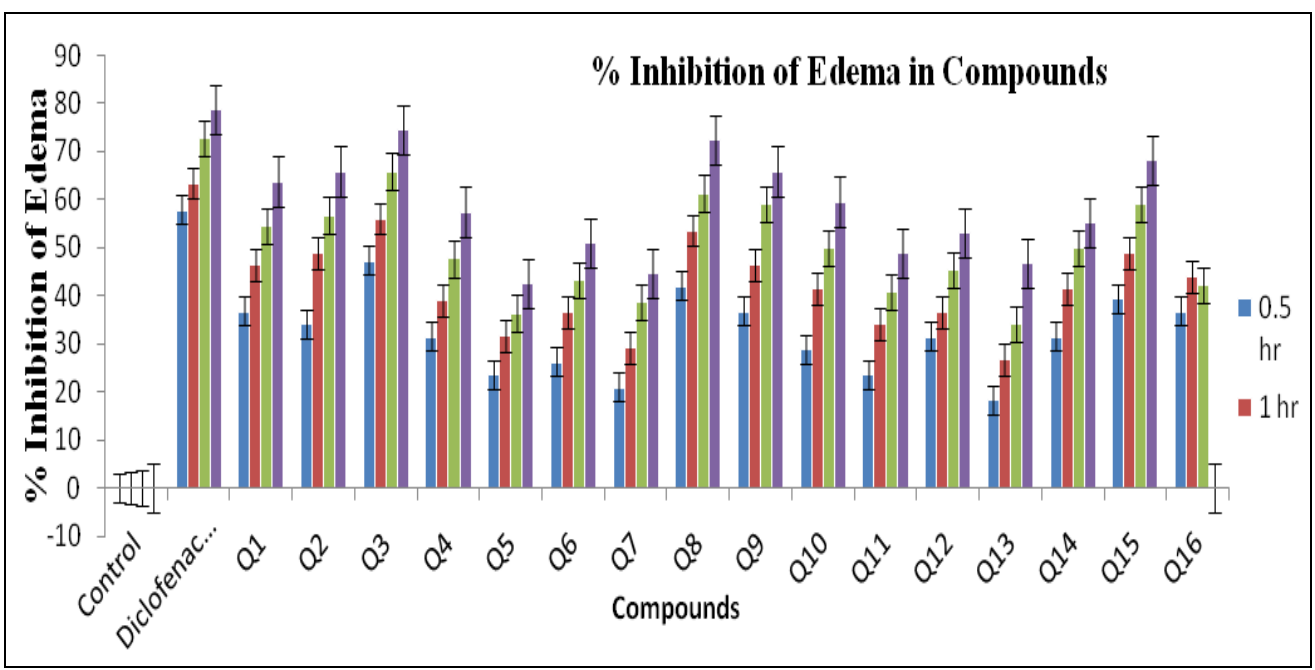

FIG. 2: EFFECT OF VARIOUS TREATMENTS ON MEAN EDEMA THICKNESS IN CARRAGEENAN INDUCED RAT PAW EDEMA 
Biochemical Estimation: The potent quinazolinone derivatives Q3, Q8, and Q15 were chosen for further biochemical estimation of cytokines and tissue lipid peroxidation.

Estimation of Cytokines Tumor Necrosis Factor Alpha, Interleukin 6 and Interleukin 1 In Serum: Synthesized quinazolinone derivatives showed good significant $(\mathrm{p}<0.01)$ and $(\mathrm{p}<0.05)$ inhibition of cytokines likes Tumor Necrosis Factor Alpha (TNF- $\alpha)$, Interleukin-6 (IL-6) and Interleukin-1 (IL-1) compared to control group.

As shown in Table 4, there was a dose-dependent decrease in all three serum cytokines (TNF- $\alpha$, IL-6, IL-1) in Q3, Q8 and Q15 groups at both $100 \mathrm{mg} / \mathrm{kg}$.

TABLE 4: EFFECTS OF QUINAZOLINONE DERIVATIVES ON SERUM CYTOKINES IN CARRAGEENAN INDUCED PAW EDEMA

\begin{tabular}{cccc}
\hline Groups & TNF- $\boldsymbol{\alpha}(\mathbf{p g} / \mathbf{m l})$ & IL-6 $(\mathbf{p g} / \mathbf{m l})$ & IL-1 (pg/ml) \\
\hline Control & $698.14 \pm 18.67$ & $589.12 \pm 18.07$ & $243.26 \pm 19.21$ \\
Diclofenac Sodium & $412.34 \pm 17.26 * *$ & $263.41 \pm 14.64 * *$ & $159.37 \pm 16.10 * *$ \\
$\mathrm{Q}_{3}$ & $521.14 \pm 23.55^{* *}$ & $386.37 \pm 15.12 * *$ & $157.14 \pm 13.02 * *$ \\
$\mathrm{Q}_{8}$ & $637.19 \pm 23.63 *$ & $464.07 \pm 22.84 *$ & $209.47 \pm 17.34 *$ \\
$\mathrm{Q}_{15}$ & $588.23 \pm 16.39 * *$ & $451.09 \pm 14.19 * *$ & $181.78 \pm 17.52 * *$ \\
\hline
\end{tabular}

Values are expressed as mean \pm SEM. $* \mathrm{P}<0.05, * * \mathrm{P}<0.01$. SEM: Standard error of the mean

TNF- $\alpha$ : Tumor necrosis factor-alpha, IL-Interleukin

Estimation of Tissue Lipid Peroxidation in Serum: As shown in Table 5 there were a dosedependent significant decrease in Malonylaldehyde (MDA) levels in all three groups. All three Compounds (Q3, Q8, and Q15) showed good significant $(\mathrm{p}<0.01)$ and $(\mathrm{p}<0.05)$ inhibition of lipid peroxidation (decrease in MDA) compared to the control group. The Diclofenac sodium showed $64.38 \%$, Q3 showed 45.17\%, Q8 showed 19.86\%, and Q15 showed a $31.27 \%$ decrease in MDA level in the brain. In this study, we found that titled compounds decreased the levels of MDA in serum after carrageenan injection.

TABLE 5: EFFECTS OF QUINAZOLINONE DERIVATIVES ON TISSUE LIPID PEROXIDATION (MDA) INCARRAGEENAN INDUCED PAW EDEMA

\begin{tabular}{cccc}
\hline Groups & Dose $(\mathbf{m g} / \mathbf{k g})$ & MDA $(\mathbf{n m o l} / \mathbf{m g}$ protein) & Percentage Decreases in MDA \\
\hline Control & $2 \mathrm{ml}$ & $0.81 \pm 0.049$ & 0 \\
Diclofenac sodium & 50 & $0.27 \pm 0.061^{* *}$ & 64.38 \\
$\mathrm{Q}_{3}$ & 100 & $0.48 \pm 0.501^{* *}$ & 45.17 \\
$\mathrm{Q}_{8}$ & 100 & $0.68 \pm 0.698^{*}$ & 19.86 \\
$\mathrm{Q}_{15}$ & 100 & $0.51 \pm 0.439^{* *}$ & 31.27 \\
\hline
\end{tabular}

Values are expressed as mean \pm SEM. $* \mathrm{P}<0.05, * * \mathrm{P}<0.01$. SEM: Standard error of mean MDA- Malonylaldehyde

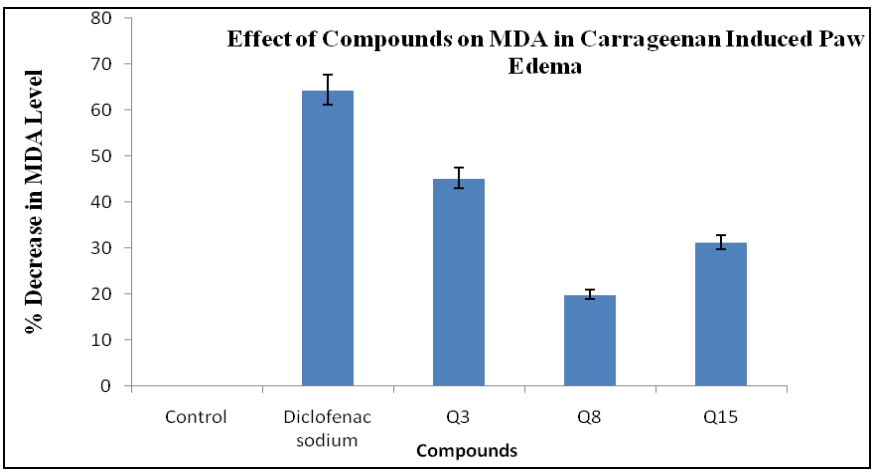

FIG. 3: EFFECT OF QUINAZOLINE DERIVATIVES ON MDA IN CARRAGEENAN INDUCED RAT PAW EDEMA

CONCLUSION: The main focus of this research work was to synthesize novel series of quinazolinone derivatives, purify, characterize and evaluate their anti-inflammatory activity. From the results, it can be concluded that the modified quinazolinone shows significant biological evaluation as anti-inflammatory agents. However, further evaluation of quinazolinone will be undertaken concerning the structural arrangements in ring for anti-inflammatory activity.

ACKNOWLEDGEMENT: The authors are thankful to the Indore Institute of Pharmacy, Indore for providing chemicals and facilities to conduct research work.

CONFLICTS OF INTEREST: The authors declared no conflict of interest.

\section{REFERENCES:}

1. Hemalatha K, Selvin J and Girija K: Synthesis, in-silico molecular docking study and anti-bacterial evaluation of 
some novel 4-anilino quinazolines. Asian Journal of Pharmaceutical Research 2018; 8(3): 125-32.

2. Yihenew Simegniew B, Adnan Ahmed B and Ariaya H: In-vivo antimalarial evaluation of some 2, 3-disubstituted$4(3 \mathrm{H})$-quinazolinone derivatives. BMC Research Notes 2015; 8: 01-06.

3. Panda S and Tripathy UP: Quinazoline a molecule of significant pharmacological and biological activity. Research Journal of Pharmacy and Technology 2013; 6(8): 849-55.

4. Kumar A, Sharma P, Kumari P and Kalal BL: Exploration of antimicrobial and antioxidant potential of newly synthesized 2, 3-disubstituted quinazoline-4(3H)-ones. Bioorganic and Medi Chem Letters 2011; 21: 4353-57.

5. Rasha SG and Mohsen MK: Synthesis and reactions of some new quinazoline derivatives for In-vitro evaluation as anticancer and antimicrobial agents. Journal of Heterocyclic Chemistry 2018; 000: 1-8.

6. Krishnan SK, Ganguly S, Veerasamy R and Clercq ED: Synthesis, antiviral activity and cytotoxicity evaluation of Schiff bases of some 2-phenyl quinazoline-4(3H)-ones. European Jour of Medicinal Chemistry 2010; 45: 5474-79.

7. Reddy PSN, Mittapelli V and Reddy VD: Antibacterial, antifungal and antifeedant activity of quinazolinonyl- $\beta$ lactams/quinazolinones and bis (quinazolinonyl- $\beta$ lactams). Rasayan Journal of Chemistry 2010; 03(04): 635-40.

8. Alagarsamy V, Chitra K, Saravanan G, Solomon VR, Sulthana MT and Narendhar B: An overview of quinazolines: pharmacological significance and recent evelopments. European Journal of Medicinal Chemistry 2018; 151: 628-85.

9. Hatem AA, Rabab DE, Mohamed M, Hassane AE, Yousreya MA, Hanan AN and Rashad AS: Molecular docking and anticonvulsant activity of newly synthesized quinazoline derivatives. Molecules 2017; 22: 01-13.
10. Alanazi AM, Abdel-Aziz AAM, Shawer TZ, Ayyad RR, Al-Obaid AM, Al-Agamy Mohamed HM, Maarouf AR and El-Azab AS: Synthesis, antitumor and antimicrobial activity of some new 6-methyl-3-phenyl-4(3H)quinazolinone analogues. Journal of Enzyme Inhibition and Medicinal Chemistry 2015; 31: 721-35.

11. Mehta S, Kumar S, Marwaha RK, Balasubramanian N, Kalavathy R, Siong Meng L, Ali Shah SA and Vasudevan M: Synthesis, molecular docking and biological potentials of new 2-(4-(2-chloroacetyl) piperazin-1-yl)- N-(2-(4chlorophenyl)-4- oxoquinazolin-3(4H)-yl) acetamid derivatives. BMC Chemistry 2019; 13(113): 1-21.

12. Elsherbiny H, Sayed EL and Khaled SM: Synthesis and anti-inflammatory evaluation of some new pyrazole, pyrimidine, pyrazolo $[1,5-\mathrm{a}]$ pyrimidine, imidazo[1, 2-b] pyrazole and pyrazolo [5, 1-b] quinazoline derivatives containing indane moiety. Polycyclic Aromatic Compounds 2019; 39(3): 1-18.

13. Tiwary BK, Pradhan K, Nanda AK and Chakraborty R: Implication of quinazoline-4 $(3 \mathrm{H})$-ones in medicinal chemistry. Journal of Chemical Biology and Therapeutics 2015; 1(1): 01-07.

14. Wasfy AAF, Mohmed NA and Salman AA: Synthesis and anticancer properties of novel quinazoline derivatives. Int $\mathbf{J}$ of Research in Pharmacy and Chemistry 2015; 5(1): 34-40.

15. Yulya M, Oleksii A, Inna N, Galina B and Sergii K: Directed search of anti-inflammatory agents among $(3 \mathrm{H}-$ Quinazoline-4-ylidene) hydrazides of N-protected amino acids and their heterocyclization products. AntiInflammatory \& Anti-Allergy Agents in Medicinal Chemistry 2019; 18(2): 1-12.

16. Abuelizz HA, Hassane AE, Marzouk M, Ezzeldin E, Ali AA and Al-Salahi R: Molecular modeling, enzyme activity, anti-inflammatory and antiarthritic activities of newly synthesized quinazoline derivatives. Future Medicinal Chemistry 2017; 9(17): 1995-09.

How to cite this article:

Patel R, Saraswat R and Pillai S: Synthesis, characterization and biological evaluation of novel quinazolinone derivatives as antiinflammatory agents. Int J Pharm Sci \& Res 2021; 12(4): 2296-05. doi: 10.13040/IJPSR.0975-8232.12(4).2296-05.

All @ 2013 are reserved by International Journal of Pharmaceutical Sciences and Research. This Journal licensed under a Creative Commons Attribution-NonCommercial-ShareAlike 3.0 Unported License.

This article can be downloaded to Android OS based mobile. Scan QR Code using Code/Bar Scanner from your mobile. (Scanners are available on Google Playstore) 